\title{
CARLOS DARWIN, UN GRAN NATURALISTA
}

\section{G. Halffer}

Red de Ecoetología

Instituto de Ecología, A.C. Carretera Antigua a Coatepec No. 35 i. El Haya

Xalapa, Ver.

E-MAIL: GONZALO.HALFFTER@INECOL.EDU.MX

A estas alturas del año Darwin, es realmente difícil escribir algo no antes mencionado. Voy a intentarlo al referirme a la obra de Darwin como conjunto, considerada como la sucesión de trabajos de un gran naturalista, reflejo y a su vez síntesis de una época dorada de la Historia Natural: el Siglo XIX. Esta visión integral recalca por qué las grandes contribuciones de Darwin contaron con un respaldo tan sólido de información (original o recopilada) y al mismo tiempo con un profundo conocimiento de las ciencias naturales de su época, dos elementos que fueron clave en su amplia difusión e impacto. Si Darwin no hubiera tenido una sólida formación como naturalista, esta visión general no hubiera sido posible.

En la segunda parte del Siglo XVIII, pero muy especialmente durante el Siglo XIX, florece un nuevo tipo de estudioso: el naturalista. A diferencia de lo que después ocurrirá en el transcurso del Siglo $\mathrm{XX}$, el naturalista aunque podía tener campos de especialización bien definidos, conservaba abierto un interés global hacia el conjunto de las ciencias naturales: la geología, la botánica, la zoología, la distribución de los seres vivos y sus relaciones con el ambiente. Un arquetipo de naturalista fue Darwin. A través de su vida trató diversos temas de Geología, Zoología y Botánica, todos ellos vertebrados por el interés muy pronto concebido y mantenido toda la vida por explicar el cambio de las especies mediante la selección natural. Su idea del gradualismo en la evolución de los seres vivos (inseparable del concepto darwiniano de selección natural), tiene una relación directa con el gradualismo en los fenómenos geológicos (concepto opuesto al catastrofismo de Cuvier y otros) que Darwin adquirió del geólogo Lyell y que modeló su interpretación de los fenómenos geológicos.

Siendo Darwin muy joven, John Henslow, profesor de Botánica en Cambridge, lo persuade de que estudie Geología, lo que haría con la orientación de Adam Sedgwick, profesor de Geología en Cambridge. Al viaje del Beagle uno de los pocos libros que lleva son Los Principios de Geología de Charles Lyell, que utilizará ampliamente.

Durante este viaje (1831-1836) va a incrementar su experiencia como Geólogo, realizando de manera 
meticulosa y metódica observaciones de formaciones, reuniendo fósiles y muestras de rocas y animales. A su regreso a Inglaterra expondrá en la Geological Society of London (de la que fue Secretario) sus estudios sobre las costas de Sudamérica, la elevación gradual de las costas de Chile y las relaciones entre los fenómenos volcánicos y la elevación de los continentes, sobre los depósitos de mamíferos fósiles de la región del río de La Plata, sobre los arrecifes coralinos, etc. En la Inglaterra de la época, Darwin va a ser considerado como un experto Geólogo. Estos primeros trabajos van a ser un antecedente a sus estudios de evolución del mundo vivo, al proporcionarle una idea de la historia de la tierra, marcada por el cambio lento y gradual a través de millones de años. Así lo reconoce en la Introducción al Origen de las Especies, así como en los capítulos dedicados a la imperfección del registro geológico y a la sucesión geológica de los organismos.

Como señala el propio Darwin, el viaje del Beagle fue importantísimo en su formación. Darwin aguantó durante cinco años las incomodidades de la vida a bordo de una fragata de la Marina Real, especialmente la falta total de intimidad y de un espacio propio, y durante cinco años fue un colector acucioso de materiales y observaciones. A su regreso, mucho del material fue entregado a especialistas para su estudio, pero la información recogida en sus diarios se ha considerado (y el mismo Darwin así lo hace) como el germen del Origen de las Especies. El viaje del Beagle va a cambiar por completo la visión del mundo natural del joven Darwin. Cabe reflexionar sobre la importancia que para las ciencias naturales tuvieron estos viajes de observación-colecta que abrieron nuevos mundos a la ciencia europea. Además de Darwin, de Humboldt, y Bonpland, Alfred Russel Wallace, con Darwin proponente de la primera versión de la evolución por selección natural y fundador de la Biogeografía moderna, realizó dos largos viajes: uno al Amazonas, el segundo al Archipiélago Malayo; Henry Bates, otro gran evolucionista y entomólogo, realizó un largo viaje al Amazonas, y así varios más.

A su regreso a Inglaterra, Darwin va a dedicarse a preparar la crónica del viaje del Beagle (1839) y los libros más especializados relacionados con el viaje: las observaciones geológicas, la recopilación de estudios zoológicos (1840), y el estudio de la estructura y distribución de los arrecifes de coral (1842). La Crónica del Viaje del Beagle por su amenidad, capacidad de observación y amor a la naturaleza ha influido mucho en el desarrollo de la vocación de posteriores naturalistas, entre ellos el que esto escribe.

En julio 1836, nueve meses después de su retorno a Inglaterra, abre su primer cuaderno de notas sobre hechos relacionados con el origen de las especies, tema sobre el que había reflexionado mucho durante el viaje y el interés por el cual va a mantener abierto toda su vida. Cabe señalar una de las características más notables de Darwin como científico: la capacidad de trabajar sobre un tema por largos años, reuniendo cuanta información encontraba publicada o de alguna manera llegaba a sus manos (mantenía una copiosa correspondencia). Esta información se iba acumulando en sus famosas carpetas que mantuvo por años y años.

Después del éxito de público que tuvieron Las Crónicas del Beagle y sus otros trabajos, de 1846 a 1854 , Darwin va a concentrar su interés en un grupo de crustáceos: los cirrípedos. Según relata en su autobiografía, durante ocho años va a trabajar constantemente con estos crustáceos, sobre los que publicará dos gruesos volúmenes describiendo todas las especies vivas que pudo examinar y otros dos libros sobre las especies extintas (publicados en 1851-53).

Para algunos comentaristas resulta inexplicable cómo alguien que ya entonces reunía notas sobre el origen de las especies, haya podido dedicar ocho años a un tema taxonómico. Para mí, es totalmente coherente: el Darwin sintetizador y proponente de teorías es una faceta del Darwin naturalista. Como lo son el Darwin observador de campo o el Darwin experimentador. Por otra parte, el estudio profundo y completo de la taxonomía de un grupo es una de las formas más directas de enfrentarse con uno de los frutos de la evolución, el que más interesó a Darwin: la especiación. Creo que no es casual que otros grandes teóricos de la evolución, entre ellos Ernst Mayr, hayan sido también importantes taxónomos.

En 1838, después de la lectura del ensayo de Malthus sobre la población, Darwin prepara un primer esbozo de la que sería su teoría de la selección natural. Por aquel entonces llevaba ya dos años 


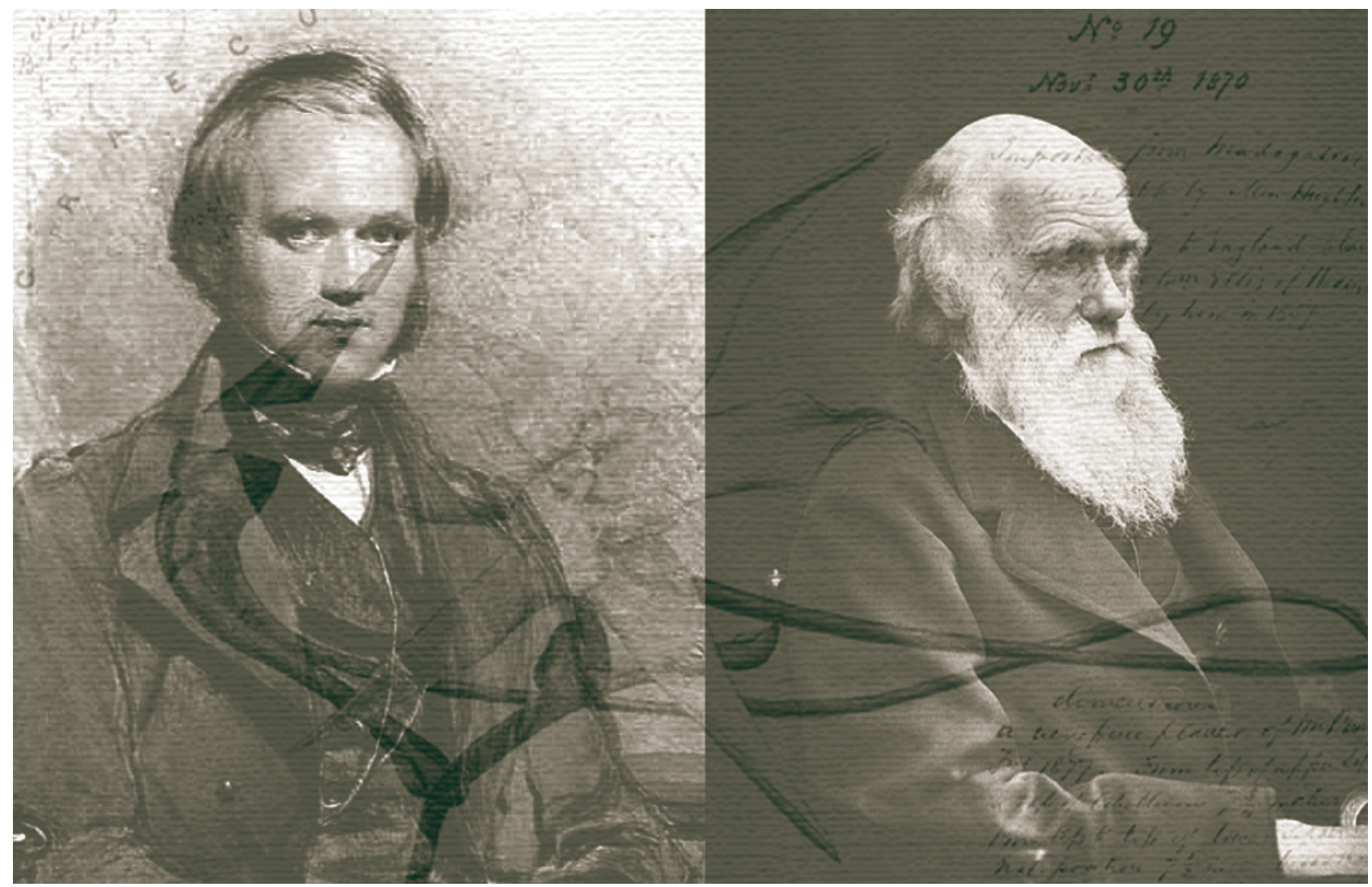

Imagen tomada y modificada de http//es.wikipedia.org

reuniendo notas sobre la evolución y la selección natural. Gracias a sus trabajos sobre los cirrípedos y a la correspondencia asociada, Darwin va a estar conectado con el mundo científico de su época. El trabajo de los cirrípedos que le había permitido ganar la medalla de oro de la Royal Society y mucho prestigio, lo sitúa como un naturalista bien conocido. Desde entonces Darwin desarrolla una de sus más notables capacidades: la lectura de una cantidad prodigiosa de bibliografía y la metódica recopilación de la información que le podía ser útil, no sólo información impresa, sino también aquella que le proporcionaban colegas e incluso horticultores, floricultores y aficionados a la cría de animales.

Por aquellos ańos, la idea de evolución estaba en el ambiente científico, pero no había sido propuesta una hipótesis coherente de cómo ocurría. Posiblemente el aporte teórico más importante de Darwin es explicar la evolución por selección natural: las variedades favorables, más adaptadas, tienden a preservarse, las desfavorables a desaparecer. Este es el mecanismo darwiniano para explicar la formación de nuevas especies: los cambios pequeños se van acumulando y originan nuevas variedades y nuevas especies. La selección natural actúa como la selección artificial manejada durante siglos por agricultores y ganaderos. Esta es una de las razones por las que Darwin mantuvo siempre un gran interés por las variedades derivadas de la domesticación y cría selectiva, a las que más tarde dedicó un libro específico.

Es bien conocido como en 1858, una carta y un estudio que le envía desde el Archipiélago Malayo, Alfredo Russel Wallace le llevan a preparar una síntesis de sus trabajos sobre evolución, la misma que se presenta en la Linnean Society junto con la comunicación de Wallace. Ambas comunicaciones van a salir publicadas en los Proceedings of the Linnean Society en 1858. El Origen de las Especies de Darwin aparecerá como libro en 1859, con un éxito inmediato. 
Los veinte años de recopilación de información transcurridos desde la preparación del primer resumen sobre la selección natural, no habían transcurrido en vano. Desde el primer momento el libro de Darwin impresiona profundamente por su potencia argumentativa y el vasto aporte de datos. Ninguna teoría científica ha logrado expandirse tanto y tan rápido en el público culto, pero no especialista. Dos años después de la aparición del Origen de las Especies, había siete ediciones diferentes en lengua inglesa, además de traducciones al alemán, francés, holandés, italiano, ruso y sueco. Al final de su vida, en los recuerdos que escribe para sus hijos (Autobiografía), Darwin señala que es un hombre rico. Además de una prudente administración de los bienes que recibió en herencia, había ganado mucho dinero con las ediciones y múltiples reediciones de sus libros. Pocos hombres de ciencia tienen con su obra impresa este éxito económico.

Es sabido que Darwin en el Origen de las Especies no hace referencia a Lamarck (si lo cita en el Origen del Hombre). Por su correspondencia y por testimonios de sus contemporáneos, se sabe que en los ańos de las grandes controversias sobre su Teoría de la Pangénesis, no le gustaba ver sus ideas asociadas a las de Lamarck. He tenido curiosidad por saber si Darwin había leído al autor francés. En su Autobiografía señala que en su juventud (antes del viaje del Beagle), leyó a Lamarck, aunque no le hizo gran impresión. Igual ocurrió con el libro de su abuelo Erasmo Darwin. Según señala en su Autobiografía, en una segunda lectura de este libro hecha hacia la mitad de su vida, Darwin encontró que contenía muy pocas observaciones y mucha especulación. Exactamente lo contrario a su propio método de trabajo.

Hacia mediados del Siglo XIX, el desarrollo de la prensa inglesa y del occidente de Europa, y la existencia de círculos culturales permitió que en torno al origen de las especies se desarrollara la primera gran polémica internacional sobre un tema científico y convirtió a Darwin en paradigma del naturalista famoso. Aunque en sucesivas ediciones del Origen de las Especies y en otras obras, Darwin contestó algunos de los argumentos contra su teoría, su aislamiento en el campo que tanto le gustaba, le permitió seguir trabajando en forma continua, aunque en torno a él la discusión entre evolucionistas y creacionistas, arreciaba. Amigos y seguidores de Darwin mucho más polémicos como Thomas Huxley, fueron los que participaron en los debates públicos. A Darwin no le gustaban las polémicas, aunque era maniático en contestar todas las cartas que recibía. Las discusiones le sentaban mal. Recordemos que desde el regreso del Beagle, la salud de Darwin fue siempre delicada. Varios comentaristas actuales coinciden en que Darwin seguramente adquirió Mal de Chagas durante sus recorridos por Sudamérica, una enfermedad grave y recurrente cuya patología era desconocida en aquellos años. La vida de Darwin en sus últimos 50 años es una alternancia de periodos de salud con otros de profundo malestar (la lectura de la Autobiografía no deja lugar a dudas). Únicamente su devoción por la investigación científica, su autodisciplina y un gran apoyo familiar le permitirán realizar su enorme obra.

El Origen de las Especies está escrito en un estilo persuasivo y fácilmente comprensible, en el que el gran acúmulo de datos lleva a las conclusiones. No es un libro abstracto. Pero así como tuvo enseguida admiradores y seguidores, también tuvo detractores. Aunque en el Origen de las Especies se hace sólo una muy lateral mención al hombre, gran parte del rechazo vino del mundo eclesiástico, ya que era, y es, imposible encontrar una conciliación entre las ideas creacionistas y la evolución de los seres vivos.

Pero hubo reacciones del mundo científico. Por ejemplo, un crítico señaló que de acuerdo a los mecanismos que proponía Darwin, se necesitarían 2500 millones de años para que el proceso evolutivo hubiera ocurrido. En aquellos tiempos la edad de la tierra se suponía de muy pocos millones de años.

La teoría de la Selección Natural ofrecía una explicación mecanicista de la evolución que no requería de un designio previo. Pero no quedaba resuelto, ni siquiera planteado, en qué forma se heredaban las variaciones. Al propio Darwin le inquietaba mucho el tema y más tarde (1868) va a proponer su Teoría de la Pangénesis, que comentamos más adelante.

El siguiente gran tema tratado por Darwin fueron las orquídeas. Como en otras ocasiones, unas observaciones de campo más o menos casuales son el inicio de su interés por el tema. Sigue la búsqueda intensiva de información y más observaciones. El 
resultado será "La Fecundación de las Orquídeas" (1862), una obra verdaderamente nueva y apasionante, donde triunfa una vez más el método de Darwin de acúmulo de información.

Durante estos años, perfecciona sus métodos experimentales para estudiar los seres vivos, que no por sencillos son menos eficaces, métodos que aplicó sucesivamente a las orquídeas, a las plantas carnívoras, a la compleja fecundación de las prímulas, a los trofismos de las plantas trepadoras y a las lombrices de tierra. Va realizando descubrimientos y experimentos iniciales al costado mismo de su casa que se revela como un mundo lleno de interacciones $\mathrm{y}$ adaptaciones.

En los mismos años, prepara nuevos libros de síntesis. En 1868 va a reunir en dos volúmenes con un total de mil páginas, sus experimentos e información amplísima sobre la variación de los animales y plantas domesticados. Una vez más Darwin es capaz de compilar una información prodigiosa, no sólo proveniente de sus propias observaciones y de publicaciones científicas, sino también y de manera muy importante de una rica correspondencia con criadores, floricultores y horticultores. Por cierto que si el Sistema Postal Inglés no hubiese sido notablemente eficiente ya en el Siglo XIX, el trabajo de Darwin en su casa de campo hubiera sido imposible.

El libro sobre los animales y plantas domesticados se inicia con una exposición de su teoría de la evolución, la que según Stephen Jay Gould es la mejor exposición breve de las principales ideas darwinianas. Si alguien quiere conocer a fondo las ideas de Darwin sobre evolución por selección, no se puede limitar al Origen de las Especies. Después de este libro y a lo largo de toda su vida, en cada nuevo libro, en cada reedición de sus obras, no sólo aporta nuevas evidencias sobre los efectos de la selección natural, también polemiza y argumenta sobre la teoría. Como ya lo hemos señalado, consolidar y expandir los conceptos de evolución por selección natural es la idea que vertebra la producción científica de Darwin.

El primer volumen del Tratado sobre las plantas y animales domesticados, es una exposición detallada y masiva de los esfuerzos realizados por el hombre para modificar animales y plantas, según sus necesidades y caprichos. El segundo volumen está dedicado a la argumentación. En la parte final de este volumen va a introducir una hipótesis sobre el mecanismo de transmisión de los caracteres: la Teoría de la Pangénesis. Darwin considera que cada parte del cuerpo de un organismo contribuye con unas gémulas a la formación de las células germinales que de esta forma aglutinan toda la información para transmitirla a la generación siguiente. Las variaciones que aparecen durante la vida de un individuo producen gémulas que así pasan a la nueva generación.

La hipótesis de la Pangénesis le da a Darwin un marco teórico que no había encontrado en el Origen de las Especies para explicar cómo opera la transmisión a la descendencia de los caracteres y sus variaciones. Varios contemporáneos consideraron la Teoría de la Pangénesis como un error de Darwin. Un intento por encontrar una explicación sin basarse en una experimentación rigurosa.

Desde sus trabajos geológicos Darwin era un gradualista. Considera la consolidación de las variaciones y la formación de las especies como fenómenos graduales que ocurren durante generaciones. Va a ser necesario llegar al Siglo XX para que se conozca la separación entre soma y germen (entre cuerpo y células germinales), así como la importancia de la mutación, y va a ser necesario llegar a la Nueva Síntesis publicada en los años cuarenta del Siglo XX, para conciliar Genética y Neodarwinismo. Es interesante señalar que uno de los principales defensores de la Pangénesis de Darwin fue Hugo de Vries, que después sería uno de los redescubridores de la obra de Mendel. De Vries acuña la palabra pangen para designar la gémula o unidad de origen. Wilhelm Johannsen va a proponer el nombre de gen en lugar de pangen.

El paradigma de evolución por selección natural se ha visto robustecido por el desarrollo de la Genética de Poblaciones y de la Biología Molecular (para una visión contemporánea véase Mayr, 1993; Gould, 2002; Pigliucci, 2007; una excelente síntesis se encuentra en Castillo-Cobian et al., 2009). La historia evolutiva de las proteínas o del ADN es la historia evolutiva de las especies; los cambios ocurren de manera constante (y generalmente gradual) y nos dan un registro directo del tiempo transcurrido (reloj molecular). 
En 1871 aparece el libro de Darwin "El Origen del Hombre y la Selección en Relación al Sexo". Ya antes varios autores, incluyendo a Lamarck, habían señalado el lugar del hombre dentro de los seres vivos. Especialmente importante es la publicación de Thomas Henry Huxley (1863): "El Lugar del Hombre en la Naturaleza”. Darwin asentó firmemente dos puntos: 1) El Homo sapiens, como las otras especies ha evolucionado de organismos precedentes por procesos naturales y bajo la presión de la selección natural; 2) El hombre comparte su ascendencia con los primates del Viejo Mundo.

El registro fósil que conocían Darwin y contemporáneos relativo al origen del hombre, era muy reducido: un antropoide (Dryopithecus) y el hombre de Neanderthal. Las evidencias para establecer las relaciones entre el hombre y los antropoides provinieron de la anatomía y embriología comparadas, muy adelantadas en la época.

El Origen del Hombre es seguido por el libro destinado a la expresión de las emociones en el hombre y los animales (1872), texto fundacional de la etología humana.

En 1875 apareció el tratado sobre las plantas carnívoras. Estas plantas según demuestra Darwin son capaces de digerir insectos y otros pequeńos animales atrapados en sus flores y hojas, y así prosperar en lugares pobres en nitrógeno y fósforo. El interés de Darwin por estas plantas se había iniciado en 1860, como siempre por observaciones de campo en las cercanías de su casa, en este caso con Drosera, género al que dedica en su libro doscientas páginas. El acúmulo de experimentos diversos, la interpretación lógica y potente, y la réplica de los experimentos por autores posteriores, va a conferir a Plantas Carnívoras una importante repercusión.

Poco después va a aparecer un largo ensayo sobre los efectos de la autofertilización en el Reino Vegetal (1876). Y en 1877, va a completar sus investigaciones sobre las estrategias de las plantas para evitar la autofecundación, con la publicación de un libro sobre las diferencias en la forma de las flores.

La última gran contribución estuvo dedicada a las lombrices de tierra y su papel en la formación del humus. Con este libro Darwin completa un artículo corto enviado a la Sociedad Geológica más de cua- renta años antes. Pero en realidad, el último libro de Darwin apareció cinco años después de su muerte. Se trata de su "Autobiografía", libro que tiene una historia interesante y muy ilustrativa.

Ya mayor, Darwin escribe los recuerdos de su vida para entretenimiento propio y de sus hijos y descendientes. Entre mayo y agosto de 1876 escribe 121 páginas, a las que en los ańos siguientes ańade otras 67.

La Autobiografía aparece publicada como parte de "Life and Letters of Charles Darwin", obra editada por su hijo Francis y publicada en 1887, reeditada en varias ocasiones y traducido a varios idiomas, entre ellos el español.

Francis Darwin, bajo la firme supervisión de la viuda de Darwin, Emma Wedgwood, introduce correcciones y sobre todo supresiones en el texto original, las más importantes en relación a las ideas religiosas de Darwin. Estas correcciones fueron motivo de mucha discusión familiar. Francis era partidario de publicar el texto íntegro, pero otros miembros de la familia se opusieron, con la idea de no provocar más a la Iglesia Anglicana y también de eliminar referencias a personajes vivos que podían resultar poco gratas. Las partes suprimidas representan el 17\% del texto original y son de un doble interés: aportan información de primera mano sobre el pensamiento de Darwin, pero también informan sobre las ideas de los censores y con ellos de una parte de la sociedad victoriana. El texto original es más vivo, más mordaz, más interesante y mucho más irónico que la versión expurgada.

Estoy hablando del texto original como si lo hubiese leído y, en efecto, lo he leído. La nieta de Darwin, Nora Barlow, recupera los cuadernos originales de la biblioteca de la Universidad de Cambridge y publica una versión completa y fiel en 1958 [Nora Barlow, The Autobiography of Charles Darwin (1809-1882), W.W. Norton and Company, 1958]. Esta versión ha sido traducida al catalán y publicada en un libro ampliamente ilustrado como monografía de la revista Mètode de la Universitat de Valencia. Para la preparación de este texto he utilizado ampliamente la publicación de Mètode.

La vida de Darwin sorprende y admira. Durante cincuenta años reunió información sobre 
el papel de la selección natural en el proceso de formación de especies, información con la que una y otra vez en distintos libros vuelve a reforzar sus argumentos. Pero además produjo a lo largo de su vida notabilísimas contribuciones sobre temas muy distintos: de los cirrípedos a la sexualidad de las plantas, a las lombrices de tierra, a la domesticación, libros en los que une la originalidad de los planteamientos al acúmulo impresionante de información. Darwin además de ser el autor del Origen de las Especies, es uno de los naturalistas más notables y productivos, quizá el más notable de todos los tiempos.

Darwin no fue un filósofo profundo, fue un observador de una extraordinaria perspicacia y de una integridad total, un trabajador incansable, con una notabilísima capacidad para reunir información y formular síntesis que son propuestas generales trascendentes. Va a alterar el curso del pensamiento no por planteamientos controversiales, sino por la profundidad de su investigación y la formación de juicios ponderados y leyes generales.

La mayor parte de las polémicas en vida de Darwin y después, han tenido como centro la confrontación con las concepciones creacionistas. Desde los 40 años Darwin era agnóstico, según él mismo se califica adoptando el término creado por Thomas Huxley. En la Inglaterra Victoriana esta manera de ver el mundo que Darwin mantuvo en sus escritos y diálogos, provocó intensas oposiciones. Todavía hoy, los sectores más reaccionarios de la Sociedad Estadounidense no aceptan las ideas darwinianas sobre evolución y mantienen para el creacionismo nuevas formas como el "diseño inteligente”, mismo que por cierto fue planteado por un contemporáneo de Darwin, el biólogo Robert Brown.

\section{NOTA:}

Una versión abreviada de este texto fue expuesta como conferencia en La Paz, Baja California Sur, en octubre 2009, a invitación del Centro Interdisciplinario de Ciencias Marinas - Instituto Politécnico Nacional. Mi agradecimiento por la invitación a mis ex alumnos muy queridos amigos, Dres. Daniel Lluch y Francisco de la Chica, así como a las autoridades del CICIMAR.

De la abundante literatura consultada quisiera resaltar, con mi muy especial reconocimiento: L'autobiografia de Charles Darwin (1809-1882). Amb restauració de les omissions que lei havia en l'edició original, a càrrec de la sera neta Nora Barlow (Traducción del original inglés al catalán por Jaume Terradas). Monografies MẼTODE, 150 pp. Universitat de Valencia, 2008. Así como el número 60 (Darwiniana) de la misma revista Mètode, invierno 2008/2009. Universitat de Valencia, en especial los artículos de Marti Dominguez, Joandomènec Ros, Jesus Purroy, José Pio Beltrán, Francisco Pelayo (en orden de paginación).

Para una visión totalmente actual de la síntesis entre Darwinismo, Genética de Poblaciones y Biología Molecular véase (la lista dista mucho de ser completa):

MAYR, E. 1993. What was the evolutionary synthesis? Trends in Ecology and Evolution, 8: 31-33.

GOULD, S.J. 2002. The Structure of Evolutionary Theory. Cambridge, Harvard University Press.

PIGLIUCCI, M. 2007. Do we need and extended evolutionary synthesis? Evolution, 61: 2743-2749.

$\mathrm{Y}$ un excelente artículo de divulgación:

CASTILLO-COBIÁN, A., A. MARTÍNEZ-A. Y A. DE LUNA-FORS. 2009. La evolución de moléculas y de sistemas biológicos. Ciencia, octubre-noviembre 2009: 22-35. 